\title{
Design and Development of Various Cloud Computing Architectures Improving the Security
}

\author{
K.Sai Manoj
}

\begin{abstract}
-numerous engineers have deliberate their very own format for introducing the distributed computing basis. The modern dispensed computing designs contrasts from severa points of view. protection has been the massive problem as patron related statistics and handling is embraced utilizing the framework gave by way of using outsider professional co-ops fluctuates especially. it's far vital to understand the degree of incorporation of security into the distributed computing framework and in some time discover the excellent layout that contains satisfactory and tight protection framework.

in this examination paper, a correlation of present models from the factor of view of attention of protection foundation internal dispensed computing framework is added along an intensive engineering that is included with each a part of protection considering.
\end{abstract}

Keywords - allotted computing, protection framework, allotted computing models, upholding protection among allotted computing frameworks

\section{INTRODUCTION}

distributed computing is essentially management version conveyed over net to gave administrations to the end clients. The principle purpose of allotted computing innovation is to assure accessibility, immoderate dependability and adaptability of infrastructural places of work which incorporate tool, programming, levels, administrations and programming which is probably appropriated to numerous registering areas. No brand new definition all subjects taken into consideration has been characterised and being pursued up 'til now. disbursed computing is a version for empowering pervasive, beneficial and an on-request set up get right of entry to to a common pool of configurable figuring belongings (e.G., memory, stockpiling, processors, set up information transmission, servers, applications, and administrations) that can be quick provisioned and discharged with negligible control exertion or professional agency connection [1]. The flighty requests of the internet 2.0 duration in combo with the craving for higher IT assets utilization are using the necessities for progressively unique IT framework that might react to fast changing necessities in an ongoing [2]. The concept of Cloud Computing is based upon on an series of numerous old and rarely any new thoughts in some research fields like provider-orientated Architectures (SOA), conveyed and matrix registering simply as virtualization [3]. In dispensed computing time, the computer can by no means once more be considered as a long way because the physical fenced in location (as an example the case, which incorporates the motherboard, the

Revised Manuscript Received on September 14, 2019.

Dr.K.Sai Manoj, CEO Innogeecks Technologies and Amrita Sai Institute of Science and Technology, Vijayawada, AP, India.

(E-mail: ceo@innogeecks.com) processor, memory, stockpiling and related components that contain the computer). alternatively the "pc" within the cloud in an ideal global includes a pool of figuring property (for example processors, reminiscence, stockpiling, and system statistics transfer ability) conceivably conveyed physical crosswise over numerous servers and geological limits, which can be looked after out on request into a very particular coherent entity(i.E. A "cloud pc") that can be grow and cringe continuously so one can assure the correct levels of state of no activity affectability, execution, versatility, unwavering tremendous and safety to any software program that unexpected spikes in call for for it [2]. therefore, cloud version is constituted of five essential characteristics, three assistance models, and four corporation fashions [1].

\section{II.METHODOLOGY}

giant concept on this exploration Paper:

special types of elements are taken into consideration right right into a allocated computing framework format which basically clarifies the way wherein the segments are organized and the manner in which the correspondence takes place among the segments. The segments considered and put in the layout encompass cloud belongings, administrations, middleware, utility and framework programming. not anything approximately the tool all subjects considered is portrayed in format. The format clarifies the houses of the product items and the connections among the articles.

The layout that clarifies the dispensed computing framework will control exceptional viewpoints that include Xaas Structurers, level adaption, organizing cloud administrations, organizing cloud segments, connection among exclusive components, communicating among the gadgets, middleware that cope with the heterogeneity that exists a number of the offering gadgets or to artwork like an agent between the client and the backend registering framework, Deploying and overseeing safety segments and to be capable check one of a kind lawful problem mainly while recognized with sending out statistics over the continents[1].

severa elements exist in the cloud foundation. facts stockpiling and recuperation is made accessible as a help the client. as regards to the records privately of the same is massive. every phase has an engineering that is incorporated with through the usage of and massive allotted computing format. severa capability gadgets which can be both strengthened on the servers or the device stockpiling gadgets are bunched to form the all

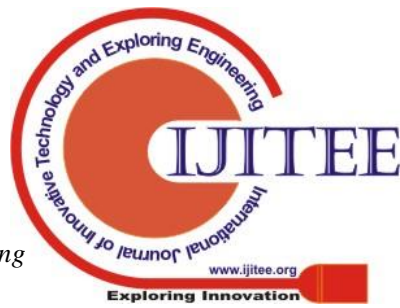




\section{DESIGN AND DEVELOPMENT OF VARIOUS CLOUD COMPUTING ARCHITECTURES IMPROVING THE SECURITY}

out capability. committed programming jogging on one of the server is made dependable to address the potential concerning task, setting away the client statistics and healing of the records in line with customer situations. The product likewise manages giving get entry to rights to the client for benefiting the statistics blessings and renouncing a comparable even as the control is finished

security has turn out to be the most tremendous hassle as regards to statistics stockpiling and restoration and transmission of the equal to forestall customers through the net. protection is likewise an issue whilst the records pass from consumer to cloud accomplished.

allotted garage no longer actually of records supported up at the server as in a conventional framework. Such distributed garage inclusive of device gadgets, stockpiling devices, servers, programs, community interface, the patron program and the framework is comparable. A subframework is executed that overseeing stockpiling in a dispensed computing circumstance

Bunched stockpiling designs are normally finished to provide efficient stockpiling and furthermore to assist adaptability and hassle tolerant records stockpiling and restoration frameworks. dispensed garage assets are usually composed as a set, framework or conveyed file framework. machine that interfaces and capacity the board programming together provide facts stockpiling administrations to the quit consumer.

offers clients easy access management and elite number one purpose facts from allocated computing framework with regards to information stockpiling and healing. ability framework gave inside the cloud computingsystems homogeneous and upheld at the level homogen on the same time as not to be the scenario now. A heterogeneous allocated garage foundation the board has been displayed [2]

Engineering has a blessing that administration considers disadvantaged as in keeping with the SLA. Engineering has been proposed relying on display off driven arrangements and virtualization improvements that help in giving adaptable allotment of administrations [3].

\section{DISCUSSIONS \& RESULT}

wherein powerfully bendy virtualization administrations gave to clients via an assistance version upheld with the resource of disbursed computing innovation. There isn't any such fashionable exists because the date for actualizing allotted computing preparations. A large amount of the engineering used to fabricate a disbursed computing framework. The necessities of the customer may be found first and in some time order every dependent on a few criteria. it's been tested that part of the highlights required via the customer, as an instance, the conditions for capability, programming, degree, records safety, and so forth., expect a massive assignment in determining the design that have to be utilized to collect cloud basis [4] processing
Investigations and findings in the cloud architectures security design point of view:

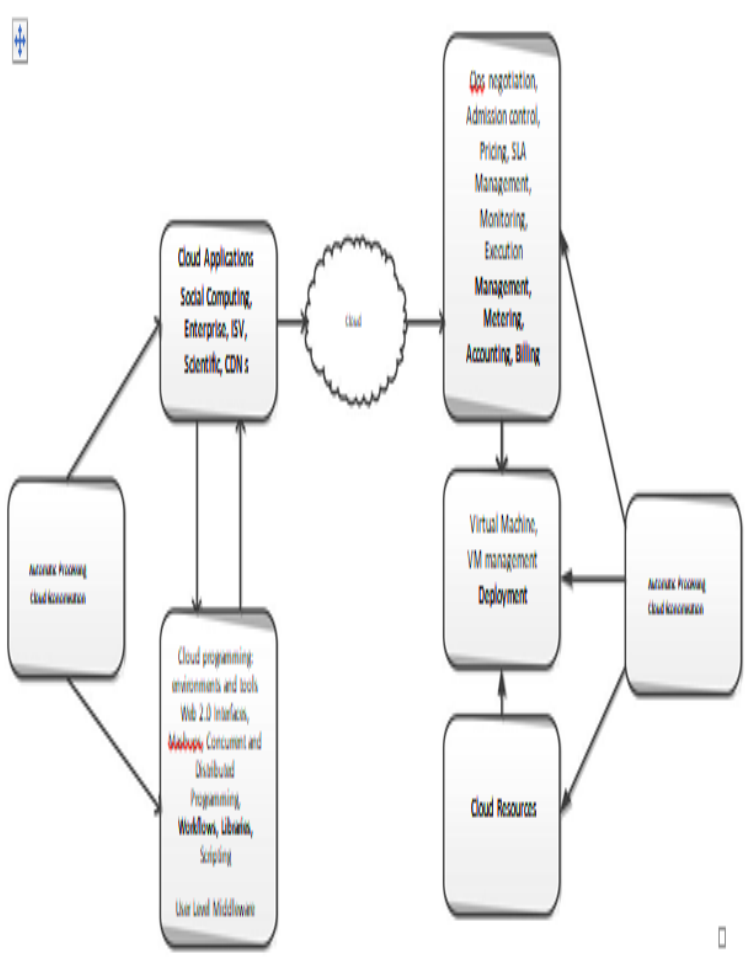

Figure 1: Related to the one way of the innovative design of cloud architecture for the improvement of the security

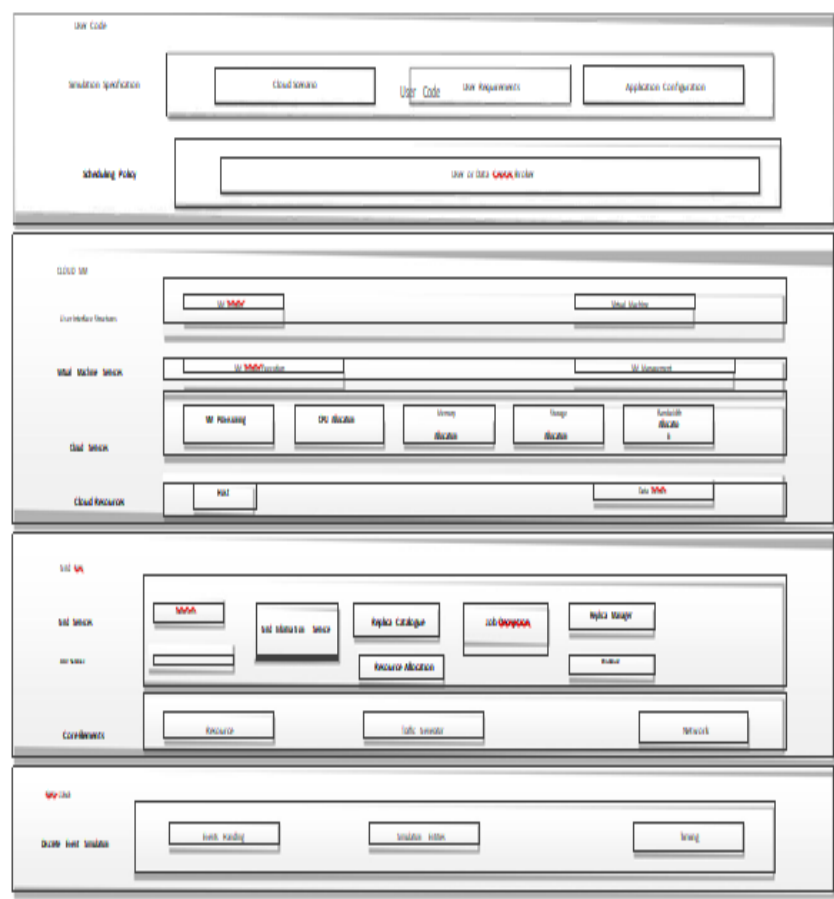

Figure 2: : Related to the one way of the innovative design of cloud architecture for the improvement of the security 


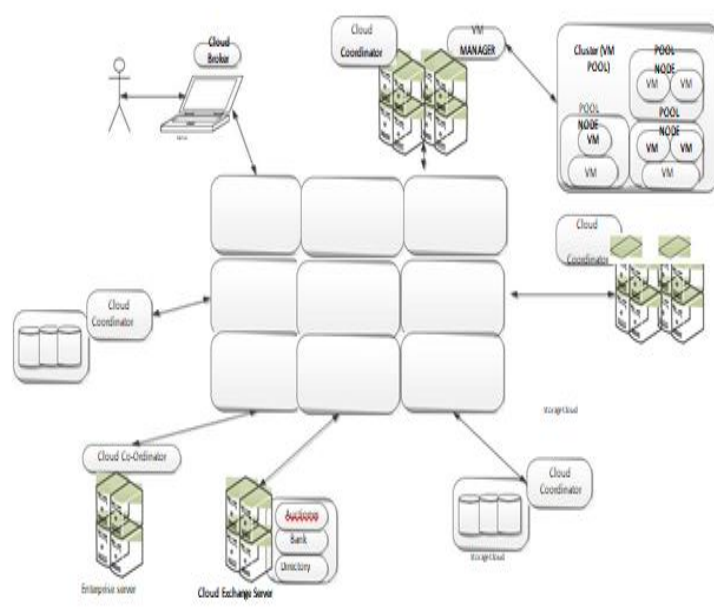

Figure 3: : Related to the one way of the innovative design of cloud architecture for the improvement of the security

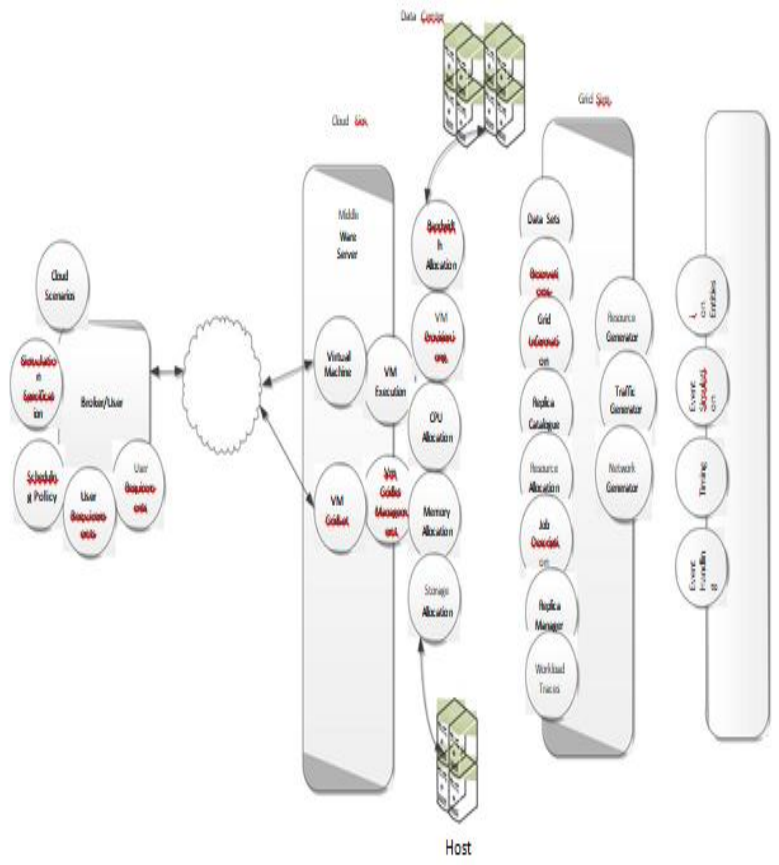

Figure 4:: Related to the one way of the innovative design of cloud architecture for the improvement of the security

\section{CONCLUSION}

on this paper, a correlation of the current layout from the viewpoint of the incorporation of the safety framework within the allotted computing framework is introduced along a miles achieving engineering that is included with each a part of protection

\section{REFERENCES}

1. Gerald Kaefer, Cloud Computing structure, corporate research and era, MunichGermany, fourth era Datacentre IEEE Spectrum, from 1 to 9.2010 .
2. Dejun Wang, An green model for Heterogeneous Cloud garage Cloud Infrastructure, magazine, 1877 7058/10.1016,510-515,2011.

3. Rajkumar Buyya1,2, Saurabh Kumar Garg1, and Rodrigo N. Calheiros, SLA-oriented aid Provisioning for Cloud Computing: challenges and solutions architecture 2011 global convention on Cloud Computing and administrations, from 1 to 10.2011 .

4. Bhaskar Prasad Rimal - AdmelaJukan - Yves Goeleven DimitriosKatsaros, Architectural necessities for Cloud Computing, journal/10.1007/DOI 10.1007/s10723-0109171-y, 1 to 26.2011.

5. Kun Liua, lengthy-jiangDonga, "Exploration information garage Cloud structure technology and Its Implementation", journal/1877-7058/10.1016,133137,2012

6. Yuri Demchenko, Canh Ngo, Marc X. Makkes, Rudolf Strijkers, 1Cees de Laat, Defining Inter-Cloud architecture for Interoperability and Integration, CLOUD COMPUTING 2012: The 0.33 worldwide conference on Cloud Computing, network, and Virtualization, ISBN: 978 - 1-61208-216-five, 1-7,2012

7. DemekeGebresenbetBayyou element IEEE1, Dr.Menchita F. Dumlao, Cloud Computing Reference structure unique in phrases of the vendor angle, global magazine of emerging technology and superior Engineering, Vol: three/problem: eleven.1 to 7.2013.

8. RajkumarBuyya, Rodrigo N. Calheiros, Jung Min Son, Amir VahidDastjerdi, and younger Yoony, softwaredescribed CloudProcessing: ArchitecturalElements and Open task, 0.33 global convention on Advances in Computing, Communications and facts technology (ICACCI), from 1 to 12.2014 .

9. A Survey of Multimedia content protection in Cloud Computing, Dr. okay.Sai Manoj, Mrudula Kudaravalli, international journal of laptop technological know-how and mobile Computing - Vol.6 trouble.eleven, November-2017, pg. 7-eleven

10. safety Cloud hazard elements and safety issues within the ebb and drift sample with Dr.k.Sai Manoj look at paper mentioned and exhibited in positioned together Scopus 2 worldwide conference with appreciate to substances, carried out Physics and Engineering (ICMAE 2018) in Indore. 2018 complaints of the most crucial traditional assembly on substances, applied Physics and Engineering. After Cancel logical paper check has been elevated to science guides organisation.

11. extent four trouble 7 eight issue 12, October 2019, ISSN 2278-3075 (on line) posted thru: Blue Eyes Intelligence Engineering and science publications (primary writer) (Elsevier Scopus) exam on improving 13.Conceptual Cloud orientated facts garage safety Dr.k.Sai Manoj worldwide journal of laptop technology and technology traits (IJCST) - volume 7 problem five, Sept.- October 2019

12. Cloud safety: hazard factors and safety problems in the present pattern Dr.k.SaiManoj international journal of Engineering and technology, science Publishing enterprise in October 2019 (Scopus)

\section{CREATOR PROFILE}

Card to say thank you

The paper is earnestly devoted to the darling Honble Secretary and Correspondent Sri. Mohan okay.Ram Garu, and Smt.k.Bhavani Amrita Devi Garu Sai Institute of

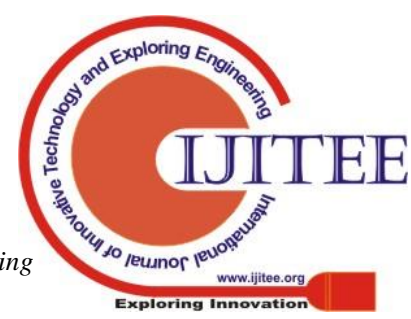


technological information and innovation. moreover to every one of the individuals from Amrita Sai appeared the executives. particular because of innovation Innogeecks, Vijayawada for his or her specialized help in all perspectives

\section{AUTHORS' INFORMATION}

Dr K Sai Manoj, Founder and Executive Director of Innogeecks Global Services Pvt Ltd, Founder and CEO of Innogeecks Technologies and Founder of 3 start-ups based on IOT and Cloud Computing, is an Enthusiastic learner, Excellent Financial Advisor, Innovative and Visionary Leader, Insightful team builder and strategic planner, who has 10+ years of experience in Financial Services, Equity Research and IT- ITeS services to his credit. He has worked in Reputed Companies like WIPRO Technologies, Fidelity Inverstments.etc.,

He is Proud of achieving many laurels in the field of Computers and Research. He is a Certified Ethical hacker, Certified Computer hacking forensics Investigator, Certified Security Analyst, Charted Engineer from IEI (India), Certified Blockchain Expert, Microsoft Certified Technology Specialist, AWS Certified Solutions Architect-Associate, Google Analytics Individual Qualification, IBM Block chain Certification, Certified EC Council Instructor and so on.

He has a proven record of having 10+ certifications from the most sought after software giants such as Microsoft, IBM, Google, Face book, EC Council \& Amazon besides this he has acted as a reviewer for the Journal of Super Computing (Springer), Journal of Big Data (Springer) and Journal of the Institution of Engineers (India) - Series B (Springer). And also with his solid financial advice 21 start-ups of Kochi, Bangalore and Vijayawada have tread the success track.

Talking about his research excellence, it is exciting to know that he has filed 3 patents and 4 more are in pipeline and has Published more than 25 research papers in reputed journals like Thomas Reuters, IEEE, Scopus etc., and shows keenness in researching on Cyber Security, Cloud Computing, Big Data Hadoop, Block chain and Data Analytics. 